\title{
Raconter la recherche-intervention. Retour sur trois opérations de gestion de la biodiversité
}

\author{
Sandrine Petit ${ }^{1}$, Philippe Fleury ${ }^{2}$, Valérie Michel $^{3}$, Catherine Mougenot ${ }^{4}$ \\ 1 Géographe, INRA, UR 718 LISTO et GIS Alpes-du-Nord, 21079 Dijon cedex, France \\ 2 Agronome, ISARA-Lyon et GIS Alpes-du-Nord, Agrapole, Département SSG, 69364 Lyon cedex 07, France \\ ${ }^{3}$ Microbiologiste, SUACI- GIS Alpes-du-Nord, 73190 Saint-Baldoph, France \\ 4 Sociologue, Université de Liège, SEED/Département Sciences et Gestion de l'environnement, 6700 Arlon, Belgique
}

\begin{abstract}
La rencontre entre des savoirs locaux, nécessairement situés, et des notions, considérées comme universelles, peut générer des conceptions innovantes qui prennent leur sens dans l'action. C'est bien ce qu'illustre ici, à l'aide de la méthode des récits, une équipe de chercheurs engagés avec le GIS Alpes du Nord dans des dispositifs d'action collective avec leurs partenaires de terrain. Il s'ensuit une production de concepts et de connaissances inédits pour chacune des parties prises séparément. La biodiversité qui est au cœur des trois cas d'étude, prend alors de nouvelles significations, diversement opératoires, mais exemplaires du sens que peut prendre une notion relative aux objets de nature, si on se donne les moyens d'un commerce d'idées entre différents types de savoirs.
\end{abstract}

La Rédaction

\section{Mots-clés :}

recherche-

intervention; agriculture ; biodiversité ; récit ;

Alpes

\begin{abstract}
Résumé - L'article se fonde sur un matériau peu courant : des récits de projets de gestion de la biodiversité associant les mondes de l'agriculture et de l'environnement. Trois situations de recherche-intervention sont ainsi analysées grâce aux concepts de la science en action. Elles mettent en évidence les caractéristiques de ces collectifs, les connaissances qu'ils mobilisent, ainsi que les outils d'intervention qu'ils construisent dans des processus mêlant recherche et action. La biodiversité dont il est question ici n'est ni celle du ministère dont c'est le domaine de compétence, ni celle des biologistes dont c'est le domaine de recherche : elle est celle dont les collectifs constitués autour de questions inédites se donnent la charge. En les suivant, grâce aux récits qu'en font les chercheurs, nous analysons les ressorts de la recherche-intervention, comparativement à une recherche plus classique.
\end{abstract}

\section{Keywords:}

intervention research; agriculture; biodiversity; story-telling; Alps

\begin{abstract}
Narratives of intervention research actions. Analysis of three biodiversity management projects. This paper is based on uncommon data: the narratives of projects dealing with biodiversity management and which associate agricultural and environmental spheres. Three intervention research situations are analysed using the concepts of science in action. The analysis is focussed on several aspects: the main characteristics of the groups including researchers and actors, the information these groups refer to, and the tools for action produced by the collective work. The kind of biodiversity which is addressed is neither the biodiversity which the Ministry is in charge of, nor the biologists' research object. It is biodiversity as defined by the groups who assume the responsibility of dealing with it. Following the group process through the researchers' narratives, we analyse the driving forces of this intervention research set-up, as compared with a more standard approach.
\end{abstract}

Auteur correspondant : S. Petit, Sandrine.Petit@enesad.inra.fr Ont participé à ce travail :

Laurent Dobremez (CEMAGREF), Emmanuel Guisepelli (GIS Alpes du Nord - Chambre régionale d'agriculture de RhôneAlpes), Raphaël Larrère (INRA), Yves Pauthenet (SUACI-GIS Alpes du Nord), qui ont aussi apporté leurs récits et contribué à l'analyse commune.

\section{Introduction}

En passant sous le vocable de biodiversité, le concept de diversité biologique a en partie échappé aux scientifiques et aux naturalistes (Aubertin et al., 1998). Discuté et manipulé par un grand nombre d'interlocuteurs, il connaît aujourd'hui une popularité croissante. Ce processus l'a 
rendu plus plastique et lui a donné des suppléments de sens, débordant des définitions classiques qui continuent à se référer aux trois niveaux, génétique, spécifique et écosystémique, de la définition de l'article 2 de la Convention sur la diversité biologique de Rio en 1992 (Barbault, 1992).

Le point de départ de cet article repose sur l'idée que ce mouvement, plaçant la biodiversité au cœur des débats de société, constitue une "transformation importante de l'action collective » (Hatchuel, 2000), susceptible de renouveler les modes de production des connaissances, en particulier, celles qui se construisent dans une posture de « recherche-intervention». Cette posture entend produire des connaissances à la fois scientifiques et utiles à l'action. Elle assume que le chercheur n'est pas «hors » mais «dans » le monde qu'il étudie et qu'il s'engage dans des situations d'interaction avec des non-chercheurs. Pour Hatchuel (2000), le recours à cette posture (qui est étroitement liée à celle des sciences de gestion) connaît des « résurgences » à certaines périodes, marquées par des «mutations importantes» et par l'émergence de nouveaux acteurs. L'élargissement des significations et des pratiques autour de la biodiversité constitue bien, pour nous, un tel moment de transition. Il invite des chercheurs de plus en plus nombreux à s'impliquer dans des projets affichant une perspective de gestion (Pinton et al., 2007). Certains chercheurs revendiquent explicitement une posture d'intervention, d'autres s'en tiennent à quelque distance, par crainte de voir leur autonomie menacée, ou encore la mettent en pratique sans y penser vraiment.

Actuellement, cette recherche tournée vers l'action associant chercheurs et acteurs est encouragée dans de nombreux programmes. En revanche, elle ne fait l'objet que de peu d'analyses et les productions auxquelles elle donne lieu restent encore mal identifiées. Jusqu'ici les études ont surtout privilégié l'angle de l'évaluation scientifique des partenariats (Hubert et Bonnemaire, 2000 ; Sébillotte, 2001), en prenant en compte les difficultés rencontrées dans leur pilotage (Soulard et al., 2007), ou encore, elles ont opté pour une perspective organisationnelle, en s'intéressant à leurs dispositifs (Hubert et al., 2004 ; Roybin et al., 2001).

Notre projet consistera, quant à lui, à suivre quelques chercheurs "impliqués » au sein du Groupement d'intérêt scientifique (GIS) des Alpes du Nord, pour tenter d'élargir la compréhension du fonctionnement des collectifs de recherche-intervention, inscrits dans le processus de changement qui s'exprime autour de la notion de biodiversité. Nous analyserons ainsi successivement : la constitution et l'autonomisation de ces groupes, la production et l'hybridation des connaissances qu'ils mobilisent et qu'ils traduisent dans des référentiels et outils de gestion. Ces processus peuvent bien entendu être regardés sous l'angle de l'action collective en cours, mais pour nous, ce sont aussi autant d'étapes qui contribuent à doter la notion de biodiversité d'un contenu et de propriétés nouvelles ${ }^{1}$.

\section{Méthodologie}

En règle générale, les articles scientifiques évitent de s'attarder sur l'identité des chercheurs impliqués et la méthodologie adoptée est censée découler le plus logiquement possible de leurs objectifs fixés a priori. Notre projet s'appuie sur des choix quelque peu différents et dont une particularité est qu'ils se sont imposés à nous chemin faisant.

Présentons d'abord rapidement la structure ayant associé les trois premiers auteurs de ce texte : le GIS des Alpes du Nord. Initié dans les années 1960, ce partenariat entre organisations de recherche (INRA, CEMAGREF, Institut technique des fromages français, Université de Savoie) et institutions de développement agricole (SUACI Alpes du Nord, Chambres d'agriculture et Conseils généraux de Savoie, Haute-Savoie et Isère) a été formalisé en 1985 par une convention. À ses débuts, le GIS abordait principalement la fonction de production de l'agriculture. Depuis 1995, les thèmes traités concernent également l'environnement, la qualité des produits et le développement territorial. Son originalité et ses modalités de travail ont déjà fait l'objet d'un article dans cette revue (Roybin et al., 2001) et elles se caractérisent avant tout par cette posture de « recherche-intervention » (Hatchuel, 2000). Dans les dispositifs mis en place par le GIS, les chercheurs recherchent et valorisent l'interaction avec les acteurs pour définir et conduire leurs travaux. Ils vont ensuite jusqu'à l'élaboration d'outils d'aide à l'action ou à une réflexion commune sur le développement.

Il faut ensuite indiquer que le matériau et la méthode utilisés ici sont peu fréquents puisqu'ils mobilisent des histoires, apportées par les chercheurs eux-mêmes. Cette expérience trouve son point de départ dans l'animation scientifique proposée par Catherine Mougenot au sein du programme DIVA (Action publique, agriculture et biodiversité) initié par le ministère de l'écologie et du développement durable. En décidant de « raconter» sur un mode familier des situations liées à la mise en œuvre de ce programme, les chercheurs ont implicitement accepté que les sujets sur lesquels ils travaillent ne se limitent pas à des objectifs définis au préalable, mais qu'ils peuvent être redécouverts au fil de l'expérience (Encadré 1 ). Dans le cas

\footnotetext{
${ }^{1}$ Ce travail réalisé dans le cadre du Groupement d'intérêt scientifique des Alpes du Nord a bénéficié des financements du programme DIVA (action publique, agriculture et biodiversité) du ministère de l'Écologie, de l'Énergie, du Développement durable et de l'Aménagement du territoire.
} 
du GIS ${ }^{2}$, l'expérience du récit a fait l'objet d'une mise en pratique durant cinq journées et a permis de revisiter un certain nombre de projets ayant eu cours de 1999 à 2006. Les chercheurs (au nombre de sept) sont ainsi devenus des narrateurs, mettant en scène la recherche-intervention et donnant à voir ses ressorts, ses parties prenantes, mais aussi ses coulisses. À l'issue de cette étape qui n'a sans doute laissé aucun des participants indifférents, ils ont sélectionné les trois récits qui leur paraissaient les plus complets et aussi les plus représentatifs des situations évoquées. Enregistrés, ces récits furent retranscrits entièrement et résumés par leurs auteurs et le stade suivant a consisté à les discuter collectivement.

$C^{\prime}$ est là que les chercheurs ont imaginé en faire une analyse plus détaillée en se référant à la sociologie de la science en action (Latour, 1989), une perspective particulièrement intéressante pour penser les interactions entre science et société, puisqu'elle présuppose qu'il n'y a pas de séparation nette entre elles. Dans la vision développée par cet auteur, l'image classique d'une recherche fondamentale cantonnée au périmètre du laboratoire isolant le chercheur et son objet de la société est en effet corrigée par son insertion au sein de cinq horizons (Latour, 1995) (Fig. 1). Dans ce modèle, la production qui est tenue pour la plus spécifique dans la recherche (la production de concepts et de théorie) est à l'intersection de quatre autres types d'actions (ou de quatre horizons différents). D'abord, il y a l'action qui consiste à «mobiliser le monde » (par le biais d'instruments, de collectes, d'enquêtes, afin de produire des données lisibles et manipulables); ensuite, il y a celle qui est à l'origine de "l'autonomisation » de la recherche (créer des "collègues », c'est-à-dire des gens capables de comprendre ce qui est dit et fait et de l'évaluer) ; puis, celle qui réussit à mobiliser les alliances nécessaires (promesses à tenir contre le recueil de financements); et enfin celle qui assure la mise en scène de la recherche (soit un travail de relations publiques capable de traduire la recherche et ses impacts dans les opinions et les pratiques). Ce modèle montre les relations qui se tissent entre le chercheur et son "contexte», autrement dit, il met en évidence les modes $d^{\prime}$ ' «attachements » du chercheur, lesquels sont généralement très peu explicités. Or, dans le cas de la recherche-intervention, ces attachements sont revendiqués d'emblée. Pour les chercheurs du GIS, l'application du modèle aux collectifs dans lesquels ils sont impliqués a donc consisté à questionner l'agencement de ces cinq horizons, avec une interrogation spécifique sur les différentes facettes du concept de biodiversité. C'est ce choix qui constitue le fil conducteur de la réflexion qui suit.

\footnotetext{
${ }^{2}$ Dans ce programme (Barre et Baudry, 2006), le projet mené par le GIS était intitulé «Biodiversité : atout et/ou contrainte de développement pour l'agriculture des Alpes du Nord?" (Larrère et Fleury, 2006).
}

\section{Encadré 1. Les ficelles du récit}

«A good story »... Et voilà la clé de tout! Aujourd'hui, autant que par le passé, les récits sont discutés, controversés et souvent même condamnés. Les récits sont indéniablement rattachés aux cultures populaires, ils manquent d'autorité... et pourtant, leur extrême sensibilité contient quelque chose de trouble, de difficile à définir, mais qui intéresse et attire.

Cette fascination repose très certainement sur leur ressort, mis en mots par Aristote, et ensuite diffusé dans le monde francophone par Paul Veyne et Paul Ricoeur. La «mise en intrigue» est ce «tenir ensemble » qui « configure » les événements en une histoire. Et le pouvoir du récit vient précisément de cette capacité à construire des agencements nombreux, soit autant de croisements entre une multiplicité d'itinéraires possibles. S'il était discipline scientifique, on pourrait dire que, "par nature ", le récit est interdisciplinaire, capable de traverser les clivages et les catégories, en intégrant dans une même « aventure » les différentes facettes, acteurs et objets d'un phénomène. En se focalisant sur les éléments les plus surprenants, le récit apporte un supplément de compréhension qui tire son intérêt des éléments contextuels, accidentels ou encore particuliers (Mougenot, 2006). À travers les associations et les oppositions qui sont mises en évidence, il produit des connaissances errantes, mais qui ont néanmoins quelque chose à nous apprendre.

En faisant le pari que le récit peut produire de nouvelles connaissances, mettre en lumière des aspects peu explorés de leur travail, des chercheurs ont volontairement accepté de raconter leur histoire, celle de leurs recherches ou encore celle des changements induits par la problématique de la biodiversité. Et en produisant de tels récits, ils ne tentaient plus vraiment d'expliquer ou de prouver ce qu'ils disaient mais ils ont suscité pour eux-mêmes et pour ceux qui les écoutaient une attitude d'éveil. En bref, ils ont pris «le risque d'inventer et de faire exister ce dont ils voulaient parler» (Stengers, 1999).

\section{Les trois collectifs mis en scène par les récits}

Les trois récits que nous avons retenus traitent des relations entre agriculture et biodiversité dans les Alpes du Nord et mettent en scène des agriculteurs, des techniciens de l'agriculture ou de l'environnement et des chercheurs.

Dans le premier récit, le collectif dont il est question appartient à une sphère agricole. Il est composé de fromagers, les «défenseurs » de la diversité microbienne des laits, de techniciens de coopératives et de services de qualité intervenant auprès des producteurs, ainsi que de représentants des filières des fromages d'appellation d'origine contrôlée des Alpes du Nord (beaufort, tome des Bauges, reblochon) et de quelques agriculteurs. Valérie Michel, microbiologiste, parfois appuyée par d'autres chercheurs (un microbiologiste, un technologue fromager, un sociologue), accompagne le groupe dans la réflexion scientifique (Michel et al., 2001). Il s'est réuni au moins deux fois par an depuis 2001. Ce collectif convoque une diversité à laquelle on se réfère habituellement peu, difficilement appréhendable par le profane, et non investie 
par les gestionnaires de l'environnement : la diversité des micro-organismes présents dans le lait de vache. À l'origine de cette question, s'inscrivent plusieurs actions entreprises depuis 1997, impulsées par les fromagers confrontés à un appauvrissement dans la composition microbienne des laits crus, modifiant les qualités organoleptiques des fromages. La volonté des filières locales est de préserver la différenciation de leurs fromages liée à leurs caractéristiques de production, dont la présence de flore microbienne fait partie. Les contraintes sanitaires actuelles et les surenchères sur ces contraintes imposées par les distributeurs de produits alimentaires rendent difficiles toute action sur cette flore. La notion de biodiversité microbienne est d'autant plus délicate à faire partager qu'elle est non visible, que l'on ne dispose que de peu d'indicateurs pour l'évaluer et que, de surcroît, la référence au « microbe » garde toujours une connotation extrêmement négative.

Le collectif évoqué dans le second récit est initié par le parc naturel régional du Haut-Jura. Sa demande, inscrite dans le cadre de mesures agroenvironnementales liées à la mise en place du réseau Natura 2000, porte sur la définition des pratiques à préconiser pour maintenir une flore diversifiée dans les exploitations agricoles de son territoire. La variété des milieux et la multiplicité des pratiques ne permettent pas d'appliquer un conseil systématique ou standard. L'adapter à la diversité des situations nécessite de se repérer à la fois dans les pratiques et parmi les milieux biophysiques. Dans ce but, un groupe d'une quinzaine de personnes qui s'est réuni tous les trimestres de 2003 à 2006 prend en charge l'élaboration d'un outil de diagnostic et de conduite des prairies associant des critères agronomiques et environnementaux. Cet outil va prendre la forme d'une classification typologique (Petit et al., 2005). Le groupe qui s'est constitué associe, aux côtés du parc naturel régional du Haut-Jura représenté par son chargé de mission agriculture, et parfois par le président de la commission agriculture-environnement, le directeur adjoint et la chargée de mission Natura 2000, des acteurs agricoles. Ces derniers sont fortement présents de par la participation de conseillers techniques des chambres d'agriculture et des syndicats de contrôle laitier de l'Ain, du Doubs et du Jura, et du Comité interprofessionnel du gruyère de Comté. Une association naturaliste prend également part aux travaux; Sandrine Petit et Philippe Fleury interviennent en tant qu'experts scientifiques.

Le troisième récit porte sur l'histoire de la rédaction du document intitulé «Alpages et prairies de montagne - Un patrimoine biologique et agricole » (Masson, et al., 2000), un texte qui s'intéresse à la fois aux espèces animales et végétales, emblématiques des Alpes (par exemple : le tétras-lyre ou le chardon bleu) et à celles maintenues par les pratiques ordinaires des agriculteurs. Ce travail a démarré sur la base d'un prétexte assez anodin : un budget est disponible au parc national de la Vanoise pour réaliser une plaquette de sensibilisation des agriculteurs à la valeur biologique des prairies. En 1999, une rencontre est organisée entre une personne travaillant au parc et Philippe Fleury, sollicité pour sa connaissance des questions agricoles; ils sont chargés de l'écriture "à deux mains » du document. Celle-ci donne lieu à la constitution d'un collectif plus large, réunissant des naturalistes (entomologiste, botaniste, ornithologue, vétérinaire), des chargés de mission du parc national de la Vanoise, des conseillers agricoles et, à certaines occasions, quelques agriculteurs membres de groupements locaux de développement agricole $^{3}$, soit un groupe d'une douzaine de personnes qui se sont engagées dans des réunions trimestrielles pendant deux ans.

\section{De nouveaux collectifs, entre alliances stratégiques et autonomisation}

L'analyse que nous faisons de nos récits met en évidence trois caractéristiques communes à ces groupes : ce sont des lieux de débat, de confrontation de connaissances et aussi de prise de décision pour l'action ; certains participants ont une identité hybride entre recherche et développement ; et enfin ces collectifs, tout comme les équipes de chercheurs décrites par Latour (1995), opèrent des formes de clôture et d'autonomisation de leurs groupes.

\section{Des lieux de débats contradictoires et inédits}

Ces collectifs composés d'une quinzaine de personnes sont hétérogènes et constituent des groupes difficilement qualifiables. Mais avant tout, ce sont de véritables lieux de débat, des espaces d'interaction et d'échanges, une caractéristique qui est ressentie par les auteurs des récits comme originale ou pionnière : ce sont des «lieux où l'on peut causer ${ }^{4}$. Pourtant, les points de vue et les connaissances qui y circulent sont divers et, en raison de la constitution des groupes, on peut aussi imaginer que les débats y sont vifs. Ces collectifs sont donc « contradictoires ", un caractère qui est lié à la mise en tension d'idées et d'intérêts discordants entre représentants de l'agriculture et de l'environnement. Leur cohésion est d'autant plus mise en question que les participants y apportent également des débats internes aux mondes auxquels ils appartiennent. Dans le premier récit, le point de vue des fromagers et celui des agriculteurs divergent. Les débats internes frôlent aussi le conflit dans le troisième récit, car

\footnotetext{
${ }^{3}$ En Savoie les groupes locaux de développement agricole restent un maillon fort de représentation des agriculteurs et d'interface avec le niveau départemental de la chambre d'agriculture.

${ }^{4}$ Les citations dans la suite du texte proviennent des récits énoncés par les chercheurs-narrateurs.
} 
les agriculteurs locaux sont opposés au projet de document commun avec le parc national de la Vanoise, alors que leurs représentants des niveaux départementaux et interdépartementaux y sont au contraire favorables : «Il y a un enjeu sur la question de l'environnement, [...] on va contre leur avis [celui du Groupe local de développement agricole de Haute-Maurienne] car il faut travailler avec eux [le Parc] ». Symétriquement, on découvre aussi des dissensions internes au parc, relatives au travail conjoint avec le monde agricole : «Il ne fallait pas montrer que c'était trop difficile au niveau du parc de la Vanoise, parce que ceux qui portaient ça, ils prenaient des risques ».

Ces espaces de discussion suscitent aussi l'émergence de sujets inédits, inattendus, difficiles à traiter comme on le verra encore par la suite. Et nous observons ici que ces débats d'idées et les incertitudes qu'ils génèrent trouvent leur origine dans le fait que des décisions sont attendues. Car ceci est un leitmotiv prégnant dans nos récits : «quoi qu'il en soit, il faut trancher!». Cette nécessité concerne autant les connaissances à retenir comme pertinentes, la définition de catégories pour les organiser, que les actions à mettre en œuvre pour gérer concrètement la biodiversité. L'issue du travail est ainsi difficilement prévisible au moment où les premiers débats s'engagent. L'imprévisibilité est d'autant plus forte que les participants peuvent endosser des rôles multiples qui ne sont pas déterminés à l'avance.

\section{Des membres à l'identité hybride}

Dans la recherche standard, les scientifiques sont les seuls à apporter des connaissances. Ici, dans les collectifs que nous suivons, ils travaillent côte à côte avec des agriculteurs, des techniciens, qui convoquent régulièrement leurs propres savoirs.

Par ailleurs, nos récits mettent particulièrement en évidence l'identité « hybride » et changeante des conseillers agricoles selon les différents moments du processus collectif. Tantôt ils se font porte-parole des intérêts stratégiques des agriculteurs : ils parlent du souci des agriculteurs d'assurer le rendement fourrager dans la gestion des prairies, même si celle-ci doit être " agri-environnementale », ou ils rappellent les contraintes sanitaires qui s'imposent dans la production laitière. Tantôt, ils agissent au titre $\mathrm{d}^{\prime}$ ' adjuvants » de la recherche : ils assurent en partie la collecte des données et fournissent une forme d'expertise de par leur connaissance de terrain. Pour les conseillers agricoles, cela revient à mobiliser leur position d'observateur privilégié des pratiques des agriculteurs (Lémery, 1994). Finalement, en accord avec leur mission classique de « relais » de connaissances (Lémery, 1994), ils ont également leur mot à dire sur les outils et les informations restitués aux agriculteurs ou à une audience plus large. À ce titre, ils sont aussi parties prenantes de la recherche dans sa «mise en scène » puisqu'ils veillent à l'accueil réservé à ces idées nouvelles. Comme le souligne Billaud (2006) pour les chargés de mission Natura 2000, le travail d'appropriation d'une nouvelle démarche de gestion de la biodiversité par les acteurs suppose l'agencement de plusieurs registres permettant l'action collective : rendre intelligible les nouvelles normes, avancer des preuves scientifiques, emporter l'adhésion et enfin changer les routines. Conscients des différentes épreuves à remplir pour inciter au changement de pratiques des agriculteurs, les techniciens évoqués dans le premier récit s'avèrent soucieux de la cohérence du conseil technique fourni dans une perspective de long terme. En effet, pour le monde technique du conseil autour du lait et de la traite, favoriser les équilibres bactériens est un changement de référence radical. Le lait devient un produit vivant duquel la flore microbienne n'est plus à éliminer systématiquement. Cela suppose de changer la nature des interventions qui, jusque-là, consistaient à généraliser des pratiques d'hygiène, voire de désinfection. Or il est difficile de dire aux agriculteurs de ne plus laver systématiquement les mamelles de leurs vaches : "Cette notion de diversité, ils ne la voyaient pas de façon aussi globale mais plutôt comme une diversité à abattre, et maintenant, on leur dit : il y a une diversité de germes à abattre et aussi des germes à favoriser ». Il leur incombe alors de trouver d'autres messages pour véhiculer les nouvelles connaissances, afin que celles-ci soient replacées dans le contexte des conseils techniques antérieurs. Dans ces collectifs, les techniciens, parties prenantes de la production de connaissances, participent donc grandement à la mise en scène des résultats, alors qu'ils restent par ailleurs aussi des « clients » destinataires des travaux et à qui on promet quelque chose.

\section{Des processus d'autonomisation}

Le deuxième horizon de la recherche «standard » consiste à « créer » des collègues, c'est-à-dire les seules personnes capables de comprendre et aussi d'évaluer les résultats produits. Cette opération est cruciale et suppose des mécanismes institutionnels de clôture, visant à rendre le collectif autonome. Or dans les groupes que nous suivons, qui impliquent aux côtés des chercheurs d'autres acteurs, une forme d'autonomisation est également nécessaire. Les récits montrent en effet qu'une grande partie du travail commun tourne autour de cette clôture qui vise à constituer un groupe de personnes avec lesquelles « on peut causer ». Mais ce processus prend ici un caractère particulier.

D'abord cette clôture se fait à double sens, c'està-dire dans les débats internes et dans ce qui en est communiqué à l'extérieur. Il faut d'un côté définir le sujet traité par le groupe, puis produire et articuler de manière singulière des connaissances scientifiques et empiriques. Et il faut, de l'autre, choisir les informations et les outils 
véhiculés à l'extérieur, une diffusion générant des risques qui doivent être collectivement acceptés, ce qui, du même coup, renforce les liens entre les personnes présentes. Ces risques sont liés au caractère pionnier des sujets abordés. Par exemple, il s'agit d'assumer des décisions de non-lavage des trayons des vaches, pour garder un ensemencement naturel du lait et en même temps, gérer le risque sanitaire. Ou bien il faut proposer des réductions de fertilisants aux dépens des rendements, pour une gestion plus souple et plus respectueuse de la biodiversité, ou encore parler de «patrimoine biologique et agricole» et pas seulement de patrimoine naturel dans un espace protégé. Le message véhiculé à l'extérieur du groupe est le résultat d'un travail et d'un soin particulier apporté à la formalisation d'énoncés transcrits dans des outils et des documents qui résultent de longues négociations (nous y reviendrons dans la dernière partie). Un tel travail nécessite ou aboutit à des formes d'exclusion. Ainsi dans la construction des typologies de prairies, l'intervention du spécialiste de la détermination des habitats se résume à une expertise externe : «La partie inventaire botanique, caractérisation des habitats, ça a été fait plus comme une prestation de service [...] On a rendu compte des données, mais la mise en débat des habitats, de la diversité des espèces, elle ne s'est pas réellement faite dans le groupe; je crois que le botaniste était invité mais il ne venait pas... ». De même, les producteurs de lait peu favorables à un conseil moins hygiéniste ou encore ceux qui sont considérés comme "peu fiables », ne sont pas sollicités dans le collectif présenté par le récit 1 . Finalement, seuls les producteurs les plus sensibles à un conseil prônant une typicité du lait cru participent.

Une autre facette de cette clôture est la mise en porte-àfaux de certaines personnes par rapport à leur institution d'appartenance, en raison des positions adoptées par le groupe et des messages nouveaux qu'il diffuse à l'extérieur. Nous découvrons, en outre, que les frontières d'une telle clôture ne sont jamais données d'avance. Elles apparaissent au fur et à mesure de l'action collective, dans un processus d'autonomisation et de cristallisation construit autour de la cohérence des connaissances prises en compte et autour du sens partagé à donner à l'action entreprise.

Enfin, les collectifs dont nous parlons sont aussi ancrés localement, ils sont «situés ». Et leur reconnaissance est d'abord fondée par et sur le crédit accordé à leur travail de terrain, défini dans leurs conditions locales. Cette caractéristique donne encore un autre sens à ce terme d'autonomisation que nous cherchons à préciser. En effet, si les connaissances produites sont complexes, subtiles et même si elles ont une portée générique, elles peinent souvent à être reconnues au-delà du cercle dans lesquels elles ont été produites. Les sujets travaillés sont souvent marginaux dans les préoccupations des communautés scientifiques et ils ne concernent qu'un petit nombre de chercheurs. C'est le cas pour la physico-chimie des laits crus, moins investiguée que les procédés de séparation des constituants du lait, ou pour les prairies permanentes qui ont fait l'objet de moins de travaux d'agronomes que les prairies temporaires. Pour les chercheurs impliqués dans ce type de collectifs, un travail supplémentaire de reconnaissance dans des publications et des réseaux scientifiques est nécessaire. En d'autres termes, cette forme d'autonomisation locale les place dans une position délicate, puisqu'ils ne sont pas dispensés par ailleurs d'obtenir une reconnaissance scientifique de leurs pairs, non impliqués dans des recherches-intervention. Cette clôture à double sens opérée par le groupe recompose alors quelque peu le modèle proposé par Latour. Nous dessinons ainsi en surimpression dans la figure 1 une ellipse qui rend compte de la constitution de liens étroits entre l'horizon des «alliances stratégiques", conclues avec les financeurs et celui de "l'autonomisation scientifique », constitué par les cercles de collègues. Pour Latour, ces deux horizons sont rarement associés de façon visible, car il peut paraître compromettant pour un chercheur de promettre des utilités fortes, en espérant une reconnaissance de son propre milieu. Pour nous, dans le cas de chercheurs impliqués dans la recherche-intervention, ces deux processus sont fortement liés du fait de la constitution hybride de collectifs qui associent des acteurs et des chercheurs travaillant dans des directions voisines et qui s'autonomisent au travers des processus de différenciations stratégique et scientifique.

\section{Connaissances scientifiques et savoirs empiriques mis en tension}

Nos récits montrent aussi que le processus d'autonomisation des collectifs de recherche-intervention repose sur la production et l'articulation de connaissances scientifiques et empiriques et sur leur traduction en outils, un point crucial qui mérite un plus ample développement.

Les connaissances scientifiques ne sont pas les seules à être mobilisées dans les opérations de rechercheintervention. Chaque acteur étant autorisé à « mobiliser le monde », des connaissances scientifiques peuvent voisiner avec des connaissances techniques et de la pratique. De cette cohabitation dépendent aussi la cohésion et la robustesse du groupe.

Dans le récit 1, l'alerte sur les risques de disparition de la typicité des fromages au lait cru vient principalement de $l^{\prime}$ « empirisme fromager » : «Il y a une prise de conscience essentiellement due aux professionnels qui transforment et qui se disent 'stop' en termes de qualité et de typicité nos fromages ne sont plus ce qu'ils étaient [...]. Ceci a aussi été montré par le monde scientifique : des expériences Inra ou d'autres ont montré que ce n'était pas qu'une simple revendication du terrain, derrière il y avait des 


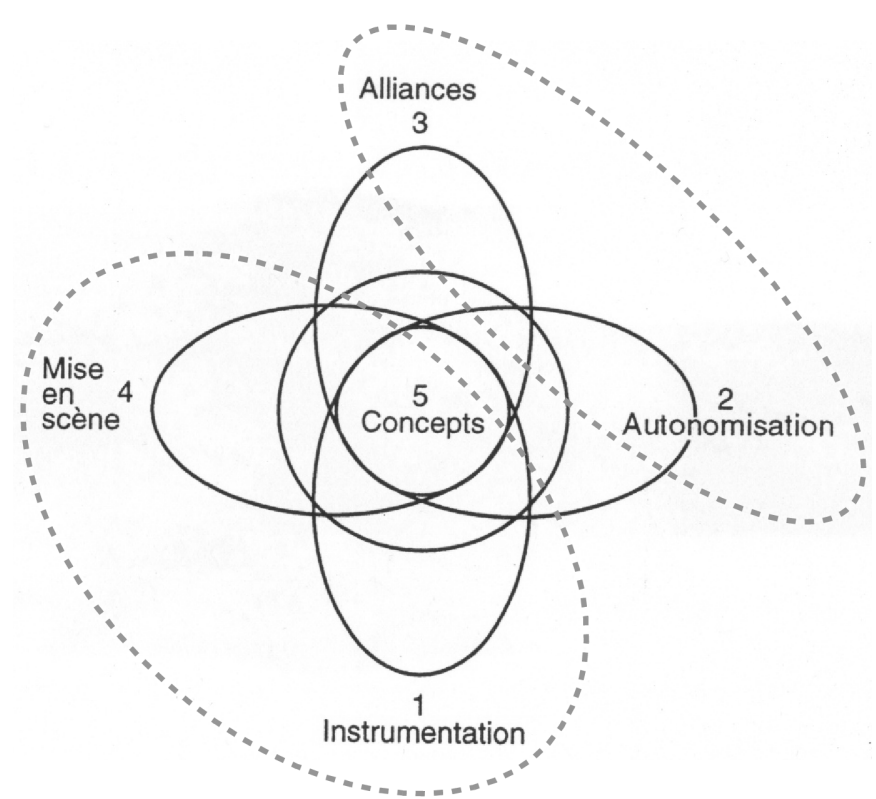

Fig. 1. Les cinq horizons de la recherche, travaillés par les collectifs engagés dans la recherche-intervention.

En trait plein, extrait de "L'intelligence sociale des chercheurs" (Latour, 1995, p. 20). En trait pointillé, les rapprochements et zones d'interactions plus intenses que nous observons dans la recherche-intervention.

Autorisation de reproduction des éditions Quae.

certitudes scientifiques ». Dans le récit 2, les savoirs des agriculteurs en matière de flore des prairies sont utilisés pour faciliter l'utilisation de l'outil typologie des prairies du Haut-Jura par les agriculteurs et les techniciens. Dans ce cas, les savoirs locaux sont mobilisés pour réduire l'écart entre les résultats scientifiques et le terrain. Que ce soit donc très en amont (récit 1) ou plus en aval (récit 2), les connaissances non scientifiques participent du processus. Notons aussi que leur absence peut être problématique. Dans le premier cas, on voit que l'entreprise sécuritaire et hygiéniste de contrôle de la qualité sanitaire du lait qui a certes conduit à améliorer la qualité globale des fromages, s'est également traduite par une homogénéisation des pratiques et, consécutivement, par la disparition d'une partie des connaissances empiriques des fromagers et des agriculteurs. Ces derniers n'ont plus les repères empiriques d'appréciation du potentiel fromager $\mathrm{du}$ lait qui leur permettaient dans le passé d'adapter leurs pratiques de transformation fromagère. Leur demander aujourd'hui de travailler un lait cru à nouveau plus riche en microbes, c'est leur demander de «mobiliser un monde » qu'ils ne connaissent plus. Cette difficulté fait écho à celle des chercheurs qui ne peuvent plus proposer un modèle d'action aussi simple et fiable que le précédent : «Dans l'interprétation des données, on peut se trouver un peu démunis pour parler de diversité à des producteurs. Je dirai démuni du fait de manque de références. Nous, on baigne dans notre ensemble de groupes microbiens avec des points de repères qu'ils [les éleveurs] n'ont pas forcément » (récit 1 ).

Les connaissances empiriques prises en compte dans les collectifs ne sont pas que des connaissances de l'action, accessoires au savoir scientifique. Elles participent du débat, c'est-à-dire qu'elles ont la capacité à entrer en contradiction avec le point de vue scientifique, qui est parfois lui-même mis en question ou montré dans ses lacunes. Dans le troisième récit, les savoirs des agriculteurs sur les relations entre leurs pratiques, comme la fertilisation, la végétation des prairies et la valeur de l'herbe pour les animaux et pour la transformation fromagère, ont été au cœur des débats pour interpeller, enrichir, et parfois même, remettre en cause les certitudes scientifiques. Ainsi, des agriculteurs ont contesté les affirmations des environnementalistes sur l'effet négatif du pâturage sur certaines espèces, telles que le chardon bleu et le trèfle des rochers. Les pratiques à préconiser ont alors été discutées différemment, par exemple la mise en défens n'a plus été le seul mode de gestion envisagé, des pâturages intermittents ont été également examinés (Larrère et Fleury, 2004).

Les collectifs, de par leur caractère hétérogène, mobilisent donc des connaissances d'origine diverse et les récits montrent qu'elles sont toutes discutables, quelle que soit leur origine. S'élabore ainsi un espace de contradiction sur la pertinence de différentes connaissances et sur leur articulation. Cette dialectique se traduit par un arbitrage. Celui-ci ne retiendra finalement que les connaissances utiles à l'action. Cette validation intervient au cours du travail du groupe et il reste difficile de prévoir quel type de savoir va finalement l'emporter : "Les catégories des biogéographes, ça n'a pas marché du tout dans l'élaboration du document et finalement, au fur et à mesure, on les a abandonnées, les savoirs des agriculteurs, des agronomes ne rentraient pas dedans » (récit 3). L'histoire montre alors que le collectif opère des choix qui lui paraissent «justes », mais seulement d'une façon locale, et qui ne seraient pas forcément validés dans un autre groupe. Ces choix sont aussi «acceptables », parce qu'ils sont rapportés à l'action collective en train de se construire.

\section{Outils et documents techniques : entre mobilisation du monde et mise en scène}

Choisir des connaissances utiles signifie aussi apprécier en amont leur capacité à être agencées sous la forme d'outils pour l'action. Dans le cas 2, l'analyse de la physionomie de la végétation utilisée dans la typologie des prairies est préférée à un inventaire exhaustif des espèces. En effet, l'indicateur de physionomie peut être relié à des niveaux de rendement fourrager, ce qui intéresse les agriculteurs. Par rapport à l'outil visé, les critères de physionomie l'emportent donc sur une description botanique. Un guide «outil de diagnostic et de conseil » présentant la typologie 
des prairies de fauche du massif du Haut-Jura a donc été édité et des formations de prise en main du document réalisées en 2006. Le premier récit évoque la mise en place d'une démarche d'accompagnement " pour un lait cru vivant ", destinée à suivre des exploitants prêts à s'engager dans une gestion fromagère du lait cru et à modifier sensiblement leurs pratiques d'hygiène liées à la traite des vaches laitières; cette démarche s'appuie sur un document technique de référence intitulé «Gestion de la flore microbienne des laits crus ». Enfin dans le dernier cas, l'ouvrage déjà cité était l'objectif même du collectif.

Dans les deux premiers récits, le travail du groupe a permis la synthèse des connaissances sous forme de typologie ou de référentiel permettant « une mobilisation du monde » par d'autres personnes que les chercheurs, que ce soit par les agriculteurs ou les techniciens. La typologie des prairies permet d'identifier un type de végétation, d'en déduire la gestion pratiquée et d'orienter les préconisations pour une gestion plus souple maintenant la diversité végétale. Dans le cas du référentiel pour les laits crus, l'éleveur devrait maximiser les germes bénéfiques qui permettront l'ensemencement naturel du lait, tout en réduisant les germes pathogènes. Il doit donc être guidé dans sa prise de décision sur l'intensité de lavage des mamelles qu'il doit pratiquer en fonction de l'état de propreté des trayons et des conditions environnementales extérieures. L'enjeu du référentiel est comme le dit l'auteure du récit de : «trouver les moyens de leur dire : ceci peut être un indicateur, ceci peut être une façon d'amplifier la diversité ». Ces outils qui traduisent les connaissances produites dans le groupe ont capacité à «mobiliser le monde » d'une manière autre que celle utilisée dans les activités scientifiques d'instrumentation ou d'enquêtes. La «mise en scène » dans les outils n'est pas seulement une activité de relations publiques. Elle amène à faire des choix dans les connaissances mobilisées et a aussi des conséquences en amont sur l'instrumentation du monde : certaines mesures ou les sujets de certaines enquêtes sont d'emblée pensés dans cette perspective.

Entre « mise en scène » et " mobilisation du monde », ces outils se lisent comme des objets complexes et hybrides. «Objets intermédiaires » (Vinck, 1999), ils jouent également un rôle actif dans la construction de partenariats qu'il s'agisse de schémas, de cartes (Mougenot et Stassart, 2008), ou comme ici de référentiels techniques (Petit et al., 2004) : "J'ai vraiment été interpellée, surprise par l'enthousiasme des gens autour de la table. [...] de voir qu'effectivement, chacun trouvait intérêt à cette typologie, $\mathrm{s}^{\prime}$ impliquait vraiment dans la construction de l'outil. Et que finalement cet outil, outre apporter des connaissances, il avait vraiment la capacité à faire de la négociation et à créer des échanges entre ces partenaires » (récit 2).

Dans les collectifs que nous suivons, les façons de mobiliser le monde sont multiples et les connaissances qui en résultent sont tout aussi variables. Pourtant, et c'est un paradoxe, il est nécessaire de les traduire rapidement de façon simple, afin de garder ou de recréer la confiance : les outils produits sont conçus et utilisés dans ce sens. Dans les trois récits, il est frappant de constater que les auteurs rapportent des anecdotes à ce sujet. Les formulations qui sont retenues à la suite de négociations complexes doivent sonner comme des «slogans » qui peuvent attester d'un réel renversement de point de vue. Dans le troisième récit, l'auteur revient sur deux formulations : celle d'un chapitre intitulé « les agriculteurs aiment la nature » et la référence au "patrimoine agricole» dans l'intitulé même du document. Derrière ces énoncés, c'est la reconnaissance de l'agriculture dans la production de biodiversité par les professionnels de l'environnement qui est en jeu. Dans le deuxième récit, la préservation de la biodiversité est abordée de façon détournée, en montrant aux agriculteurs qu'il y a un intérêt agricole à une gestion environnementale des prairies : «Les prairies extensives ont une place dans les exploitations agricoles... ». Enfin, les expressions qui émaillent le premier récit sont parlantes : «Le lait propre est du lait mort », il faut passer à un lait « riche et vivant».

Toutes ces formulations simples, trop simples sans doute aux yeux des chercheurs qui ne font pas d'intervention, accompagnent des problèmes posés de façon complexe et subtile, mais contrairement aux faits avérés scientifiquement qui peuvent rester très confidentiels, elles sont destinées à intéresser beaucoup de gens. Ces slogans participent encore à l'autonomisation du collectif en explicitant sa posture. Ils sont à la fois de l'ordre de la " mise en scène », destinés à être diffusés et à intéresser une audience large, mais ils peuvent aussi susciter de nouvelles alliances. Ils peuvent enfin tenir le rôle des publications dans la recherche classique, c'est-à-dire signifier les basculements de paradigmes. Leurs formulations témoignent de ruptures de stratégies par rapport aux modèles de développement dominants et suggèrent des changements de discours : «On avait des craintes, que la typologie servent à intensifier les prairies [...], on se pose la question finalement du conseil technique en exploitation, par rapport à quel modèle de développement le définir, quels modèles de développement pour le territoire du Haut-Jura ? Si on est plus clair là-dessus, on peut être plus clair sur quelle utilisation de la typologie de prairies dans le conseil technique » (récit 2). Ces formulations parlantes rendent compte d'un tournant validé par le collectif «Il y a un changement de discours [...] il y a même un changement de vocabulaire [...] c'est un changement de cap » (récit 1).

Mis en scène et circulant auprès de différents publics, ces outils sont comme des " publications » circulant à travers tous les horizons de la recherche-intervention pour exprimer le concept de biodiversité tel qu'il a été recomposé. Dans la figure 1, nous matérialisons une seconde ellipse montrant les interactions particulières entre les 
horizons 1, 4 et 5 . Elle souligne que dans la rechercheintervention, les scientifiques ne sont pas seuls à recueillir des données grâce à leurs protocoles de recherche. Ils s'associent à d'autres membres du collectif (agriculteurs ou techniciens, selon les formes de mobilisation qui leur sont propres, observations et tests par la pratique). Toutes ces «mobilisations du monde » sont mises en tension et traduites dans des outils qui cristallisent le travail du groupe, ses arbitrages en termes de concepts (horizon 5) et ses accords exprimés dans des messages sous forme de «slogans ». L'horizon 4, celui de la «mise en scène » joue donc ici un rôle crucial, en aval du travail du collectif (les outils élaborés permettent une nouvelle mobilisation du monde par les gestionnaires de la biodiversité), mais aussi en amont (il définit le contexte historique et local des sujets à débattre).

\section{Conclusion : «cette biodiversité dont on a la charge... »}

Notre travail a trois particularités. D'abord il est basé sur des récits qui ont permis à leurs auteurs de collecter les composantes des situations de recherche-intervention dans lesquelles ils sont impliqués. Composantes diverses, parfois même anecdotiques, mais qui nous ont paru particulièrement aptes à montrer les interactions entre science et action, soit entre les cinq horizons de la recherche proposés par Latour. Ceci a permis d'explorer d'une nouvelle manière le travail des chercheurs impliqués dans les collectifs de gestion de la biodiversité. Et pour finir, nous tentons d'en synthétiser l'essentiel en cinq points.

\section{Sujets nouveaux}

Les sujets sur lesquels travaillent les chercheurs et les acteurs que nous suivons sont peu répertoriés, peu considérés, presque marginaux. Ils ne parlent pas tant de bactéries du lait, d'espèces et d'écosystèmes à protéger, que de lait vivant ou de prairies extensives. Or ces sujets sont réexplorés, à la fois par la perspective de gestion opérationnelle et locale dans laquelle ils sont inscrits, mais aussi par l'épaisseur historique des questions qu'ils traitent. Ainsi, même s'il s'agit de biodiversité dans les débats que nous suivons, il n'est pas possible pour les participants de se passer des références antérieures, au modèle de développement par exemple, ou au conseil technique agricole. Recomposés, ces sujets vont s'éloigner des concepts scientifiques aux définitions arrêtées, ils sortent du champ de l'écologie et requièrent alors un basculement dans les idées. Ce sont des sujets "échevelés » et "risqués », pour reprendre les expressions de Latour (1999), car ils obligent à considérer des relations multiples et subtiles, dessinant des chaînes d'interdépendance, n'ayant de cesse de s'allonger
(Mougenot et Roussel, 2005). Ces relations prennent en compte des recommandations normatives, autant que des coûts de production ou des expressions identitaires. De ce fait, elles mettent en évidence un niveau d'organisation de la biodiversité plus englobant ou plus complexe que ceux des espèces ou des écosystèmes.

\section{Connaissances hybrides et en tension}

D'un concept formalisé en termes biologiques, ou d'une définition émanant des autorités qui en ont la prérogative, la notion de biodiversité dont il est question ici devient alors un mélange de pratiques, de jugements de valeur apportés par les uns et les autres, mais aussi un mélange de connaissances scientifiques ou empiriques, générales ou locales. Dans tous les cas, la biodiversité prend en compte un nombre accru de composantes, biologiques, techniques et sociales et qui, de ce fait, rapprochent presque toujours des intérêts a priori discordants. Nos récits sont émaillés de nombreuses références à des paris jamais gagnés d'avance, à des conquêtes toujours à faire dans les débats, mais aussi dans la validation des décisions à prendre. Ce faisant, ils mettent en évidence cette mise en tension entre les différentes formes de connaissances mobilisées mais aussi la mise en tension entre la recherche et l'action.

\section{Clôture fragile et risquée}

Les collectifs d'acteurs qui se constituent autour de ces sujets ont alors devant eux une tâche cruciale qui vise la viabilité du collectif. Il s'agit là en réalité « d'intéresser et de tenir son monde » (Latour, 1995). Or ceci suppose de clôturer les groupes au sein desquels les problèmes pourront être définis, débattus, évalués et critiqués, ainsi que les sujets qui pourront être pris en compte. En d'autres termes, cette tâche suppose l'inclusion ou l'exclusion de certains membres présents au départ ainsi que le choix des questions traitées ou écartées. Cette clôture est nécessaire pour rendre le groupe autonome, mais elle reste éphémère, puisqu'elle repose sur un travail de cohésion toujours à faire et sur des attachements institutionnels ou professionnels risqués pour les participants. Le travail de clôture renforce et stabilise le contenu attribué à la notion de biodiversité, mais il la montre aussi dans sa fragilité, c'est-à-dire liée à un processus toujours en évolution.

\section{Production d'outils opérationnels}

Les collectifs dont nous racontons l'histoire se caractérisent aussi par le fait qu'ils cherchent à traduire leurs débats et leurs décisions dans des outils qu'ils souhaitent (un peu) plus pérennes qu'eux-mêmes. La typologie des 
prairies, outil mis au point dès 1995 dans les Alpes du Nord avant d'être réinvesti dans le Jura, constitue un exemple d'objet qui a dépassé la durée de vie du collectif qui l'a créé. Il n'en va pas de même de l'ouvrage sur les prairies et les alpages, qui n'a pas réellement servi de référence pour d'autres actions dans les espaces protégés alpins. Le référentiel de gestion de la flore des laits dont nous parlons est, quant à lui, en train d'être testé. Ces objets sont donc eux aussi des paris jamais gagnés, et pourtant, ils ont la charge de stabiliser, de transposer les choix opérés ainsi que les connaissances récoltées et articulées. La production de ces objets reste une épreuve dont le succès peut contribuer à élargir le cercle des personnes intéressées et à dépasser la durée de vie limitée des projets dans lesquels ils ont été construits.

\section{Mise en mots}

Enfin, les collectifs que nous suivons ont également un rôle d'énonciation qui est décisif. Des énoncés peuvent faire l'objet de négociations parfois longues et subtiles, mais ils doivent aussi pouvoir sonner simplement, comme des slogans. Longs ou courts, ces énoncés vont permettre à la biodiversité, ordinairement conçue comme naturelle, biologiquement définie, de devenir aussi agricole, au sens productif, économique et social de ce terme. Car ici la biodiversité peut devenir utile, utilisable et utilisée. Pour les agriculteurs, elle peut être le support d'une élaboration nouvelle de sens et de rationalité de leurs pratiques. Cette biodiversité ne sera plus seulement une contrainte, imposée de l'extérieur, mais aussi un atout dont ils pourraient se saisir, notamment pour se démarquer d'autres systèmes de production moins précautionneux vis-à-vis l'environnement (Larrère et Fleury, 2006). Pour tous les participants au collectif, la biodiversité qui était au départ floue ou, au contraire, exclusivement cernée par une définition scientifique, devient au cours du processus, «cette biodiversité dont on a la charge ». Ce travail de mise en mots atteste de la qualité de l'interaction entre chercheurs et acteurs engagés ensemble dans une gestion dynamique et évolutive et il contribue quelque peu à en atténuer les risques.

\section{Références}

Aubertin, C., Boisvert, V., Vivien, F.-D., 1998. La construction sociale de la question de la biodiversité, Natures Sciences Sociétés, 6, 1, 7-19.

Barbault, R., 1992. Écologie des peuplements. Structure, dynamique et évolution, Paris, Masson.

Barre, V., Baudry, J., 2006. Action publique, agriculture et biodiversité. Résultats scientifiques et recommandations. Rapport, Ministère de l'Écologie et du Développement durable, INRA, Paris Rennes.
Billaud, J.-P., 2006. Ce que faciliter veut dire. À propos d'un retour d'expérience de chargés de mission Natura 2000, in Rémy, J., Brives, H., et Lémery, B. (Eds), Conseiller en agriculture, Dijon-Paris, Educagri - INRA éditions, 205-219.

Hatchuel, A., 2000. Intervention Research and the Production of Knowledge, in Cerf, M., Gibbon, D., Hubert, B., Ison, R., Jiggins, J., Paine, M., Proost, J., Röling, N. (Eds), Cow up a tree. Knowing and Learning for Change in Agriculture. Case studies from industrialised Countries, Paris, INRA éditions, 55-68.

Hubert, B., Bonnemaire, J., 2000. La construction des objets dans la recherche interdisciplinaire finalisée : de nouvelles exigences pour l'évaluation, Natures Sciences Sociétés, 8, 3, 5-19.

Hubert, B., Moulin, C.-H., Roche, B., Pluvinage, J., Deffontaines, J.-P., 2004. Quels dispositifs pour conduire des recherches en partenariat? L'intervention d'une équipe de recherche au Pays Basque intérieur, Économie rurale, 279, 33-52.

Larrère, R., Fleury, Ph., 2004. Malentendus, incompréhensions et accords dans la gestion de la biodiversité, Fourrages, 179, 307-318.

Larrère, R., Fleury, P., 2006. Biodiversité : atout et/ou contrainte de développement pour l'agriculture des Alpes du Nord?, in Baudry, J., Barre, V. (Eds), Action publique, agriculture et biodiversité. Résultats scientifiques et recommandations. Rapport, ministère de l'Environnement et du Développement durable - INRA, Paris-Rennes, 59-66.

Latour, B., 1989. La Science en action, Paris, La Découverte.

Latour, B., 1995. Le métier de chercheur regard d'un anthropologue, Paris, INRA Éditions.

Latour, B., 1999. Politiques de la nature. Comment faire entrer les sciences en démocratie, Paris, La Découverte.

Lémery, B., 1994. Une position d'expert incertaine : les conseillers techniques en agriculture, in Darré, J.-P. (Dir.), Pairs et experts dans l'agriculture. Dialogues et production de connaissances pour l'action, Ramonville, Éditions Erès, 91-116.

Masson, N., Fleury, P., Plaige, V., 2000. Alpages et prairies de montagne. Un patrimoine biologique et agricole. Parc national de la Vanoise - SUACI Alpes du Nord, Chambéry.

Michel, V., Hauwuy, A., Chamba, J.F., 2001. Raw cow milk microflora: diversity and influence of conditions of production, Le lait, 81, 5, 575-582.

Mougenot, C., 2006. Les ficelles des récits. Rapport, ministère de l'Écologie et du Développement durable, Paris.

Mougenot, C., Roussel, L., 2005. To poison or to trap? The ecologisation of 'pest' control, Sociologia ruralis, 45, 1-2, 115 129.

Mougenot, C., Stassart, P., 2008. Les objets éphémères du développement durable - Un mécanisme de représentation/tranformation, in Mélard, F. (Dir.), Ecologisation, Objets et concepts intermédiaires, Bruxelles, Peter Lang, 73-96.

Ost, F., 1999. Le temps du droit, Paris, Odile Jacob.

Petit, S., Fleury, P., Vansteelant, J.Y., Plaige, V., 2004. Les typologies de prairies, d'un outil agronomique à un objet de médiation entre agriculture et environnement, Fourrages, 179, 369-382.

Petit, S., Fleury, P., Vansteelant, J.Y., 2005. Agriculture, prairies de fauche et environnement dans le Massif jurassien. Outil de diagnostic et conseil. Guide technique, parc naturel régional du Haut-Jura - GIS Alpes du Nord, Lajoux - Chambéry.

Pinton, F., Alphandéry, P., Billaud, J.-P., Deverre, C., Fortier, A., Géniaux G., 2007. La construction du réseau Natura 2000 en France, Paris, La Documentation française. 
Roybin, D., Fleury, P., Béranger, C., Curtenaz, D., 2001. Conduites de recherches pluridisciplinaires en partenariat et apprentissages collectifs. Le cas du GIS Alpes du Nord, Natures Sciences Sociétés, 9, 3, 16-28.

Salmon, C., 2007. Storytelling: Une machine à fabriquer des histoires et à formater les esprits, Paris, La Découverte.

Sébillote, M., 2001. Les fondements épistémologiques de l'évaluation des recherches tournées vers l'action, Natures Sciences Sociétés, 9, 3, 8-15.

Reçu le 17 octobre 2007. Accepté le 26 juin 2008.
Soulard, C. T., Compagnone, C., Lémery, B., 2007. La recherche en partenariat : entre fiction et friction, Natures Sciences Sociétés 15, 1, 13-22.

Stengers, I., 1999. Le développement durable, une nouvelle approche, Alliage, 40, 31-39.

Vinck, D., 1999. Les objets intermédiaires dans les réseaux de coopération scientifique, Revue française de sociologie, 40, 2, 385-414. 